\title{
Models for Strategic Planning: Applying TBIM to the Montreux Jazz Festival Case Study
}

\author{
Fabiano Francesconi \\ Teralytics AG, Switzerland \\ fabiano.francesconi@gmail.com
}

\author{
Fabiano Dalpiaz \\ Utrecht University, the Netherlands \\ f.dalpiaz@uu.nl
}

\author{
John Mylopoulos \\ University of Trento, Italy \\ john.mylopoulos@unitn.it
}

\begin{abstract}
The Tactical Business Intelligence Model (TBIM) is a language for modeling business tactics. TBIM models act as a bridge between the strategic and tactical organizational levels, for the modeled tactics refine an organization's strategy, while needing further details to become operational. The purpose of TBIM models is to support decision making by representing and enabling the comparison of alternative plans for fulfilling an organization's strategic objectives. In this paper, we report on our experience in applying TBIM for modeling the Montreux Jazz Festival organization case study from the literature. In addition to showing TBIM models for different aspects of festival organization (ticketing and advertising, experience delivery, concerts and recordings), we discuss lessons learned.
\end{abstract}

\section{INTRODUCTION}

Decision making in organizations is a complex activity, typically handled by refinement into smaller tasks at different levels of abstraction (strategic, tactical, and operational). The strategic level is of primary importance, for it defines the longterm objectives of the organization in terms of vision, mission, and goals. Once the strategy is set, the decision to be taken concerns how to realize it in terms of viable plans.

This decision making is conducted via strategic planning [1], [12] activities, which lead to high-level business tactics whose implementation is expected to realize the strategy. The success of this activity depends on the expertise of the decision makers, the adoption of best practices, a successful realization of the tactic in the organization, also the modeling and analysis of key aspects of a business tactic.

In previous work [6], we have proposed the Tactical Business Intelligence Model (TBIM) language for modeling and reasoning about strategic plans. The language enables the modeling of business tactics in terms of ways for achieving a set of business goals through value propositions, market segments, distribution channels, production and delivery activities, as well as partnerships. TBIM extends the Business Intelligence Model (BIM) [11] for strategic modeling with primitives from the Business Model Ontology (BMO) [17].

In this paper, we report on our experience with the TBIM language in modeling the Montreux Jazz Festival organization case study, drew from Osterwalder's doctoral dissertation [17]. We show some of the models that we have created representing three aspects of festival organization: ticket selling and festival advertising, delivery of an exceptional experience to the festival audience, and organization of concerts and recordings.

978-1-4673-6630-4/15/\$31.00 (c) 2015 IEEE
We illustrate the distinguishing features of business tactics (using the tactical view of TBIM) and highlight the network of partnerships that contribute to the realization of these tactics (using the partnership view of TBIM). We discuss the benefits of our models as well as their limitations.

Organization. Our research questions and method are shown in Section II. Section III briefly presents the Montreux Jazz Festival case study. Section IV reviews our baseline, the TBIM language. Section V shows TBIM models for the case study. Section VI reports on benefits and limitations from our modeling. Section VII discusses related work, while Section VIII concludes.

\section{Research Questions And Method}

As part of the demonstration step of the Design Science Research Methodology [19], we are interested in studying the applicability of the TBIM language to large case studies, and, specifically, we investigate its potential in terms of adequacy and richness of the modeling primitives and supported models. This is summarized by the following research question:

RQ: What is the adequacy of the modeling primitives and models of TBIM for large-scale settings?

Note that our question does not study the practical applicability of the language. The presented research is a necessary, preliminary step prior to experimentation in the field, for we aim to provide modelers with rich and expressive conceptual modeling languages, before compromising their conceptual soundness in favor of usability (a key area for future research).

We split our research question into two complementary sub-questions that focus on strengths and weakness of TBIM:

SRQ1: What are the strengths of TBIM that make it adequate for large-scale settings?

SRQ2: What are the limitations of TBIM that hinder its adequacy for large-scale settings?

We employ a qualitative research method, in which one of the authors of the language studies in depth a case study from the literature, and applies TBIM to such case. After the modeling has taken place, the other authors serve as a panel that review the models in order to identify strengths and limitations. This approach is certainly not exempt from limitations (see Section VI), but we deem it adequate to investigate our research question, which focuses on the adequacy of the modeling framework, rather than its ease of use. 


\section{Case Study: The Montreux Jazz Festival ORGANIZATION}

The Montreux Jazz Festival (MJF) is a world-renown festival that hosts jazz concerts in the Swiss city of Montreux on the Lake Geneva shoreline. Since its foundation in 1967, the festival has grown into a genuine international venture with an audience spanning four continents and a budget of 15 million Swiss francs. Beside the main annual festival in Montreux, licensing agreements have led to the creation of other events including The Montreux-Detroit Jazz Festival, The MontreuxAtlanta Jazz Festival, Montreux Festival On Tour and The Montreux Jazz Festival in Monaco. Until 1995 the Festival was linked with the Montreux Tourist Office.

Due to such unprecedented growth, the Montreux Jazz Festival Foundation was established, having the Festival as its main activity. At the same time Montreux Sounds was born, a fully independent incorporation that manages the rights of the huge archive of MJF recordings and clips accumulated since 1967. Nowadays, MJF is the second largest annual jazz festival after Canada's Montreal International Jazz Festival. In the 1970s, the festival began offering other musical genres in addition to jazz, such as blues and rock, featuring popular artists such as Led Zeppelin, Pink Floyd, Frank Zappa, Deep Purple and others.

The 2003 edition of the MJF organization, has been extensively analyzed by Alexander Osterwalder in his doctoral dissertation [17]. That edition is still remembered as one of the most exceptional one because of the temperatures and an attendance of roughly 240,000 visitors. The numbers tell of a festival of more than 94,300 tickets sold, the participation of 44 DJs, 326 bands, and the involvement of 1,200 staff.

Osterwalder presents the MJF case study as an illustration of his Business Model Ontology using an early version of the Business Model Canvas. Our purpose here is to reconstruct these models using TBIM. A key advantage of our models is that they are formal, allowing automated reasoning, e.g., extensions of those proposed for the BIM language [11].

\section{The Tactical Business Intelligence Model (TBIM)}

The Tactical Business Intelligence Model (TBIM) [6] is a language for modeling and reasoning about the business tactics that are created during strategic planning. The language links the strategic and the tactical level, by providing modeling support to represent high-level business tactics. TBIM extends the BIM strategic modeling language [10], [11] with primitives for business model design taken from BMO [17]. TBIM proposes concepts that business users are familiar with, including actor, strategic goal, task/process, distribution channel, resource, etc.

The underlying BIM language is a goal-oriented language that (see Fig. 2) refines strategic goals of the organization through AND/OR links into other elements: processes within the organization (tasks), and conditions that have to hold true in the studied context (domain assumptions). Moreover, BIM includes internal and external (depending on their controllability by the organization) situations which occurrence may influence positively or negatively elements in the model such as goals and processes. Finally, indicators are metrics that can

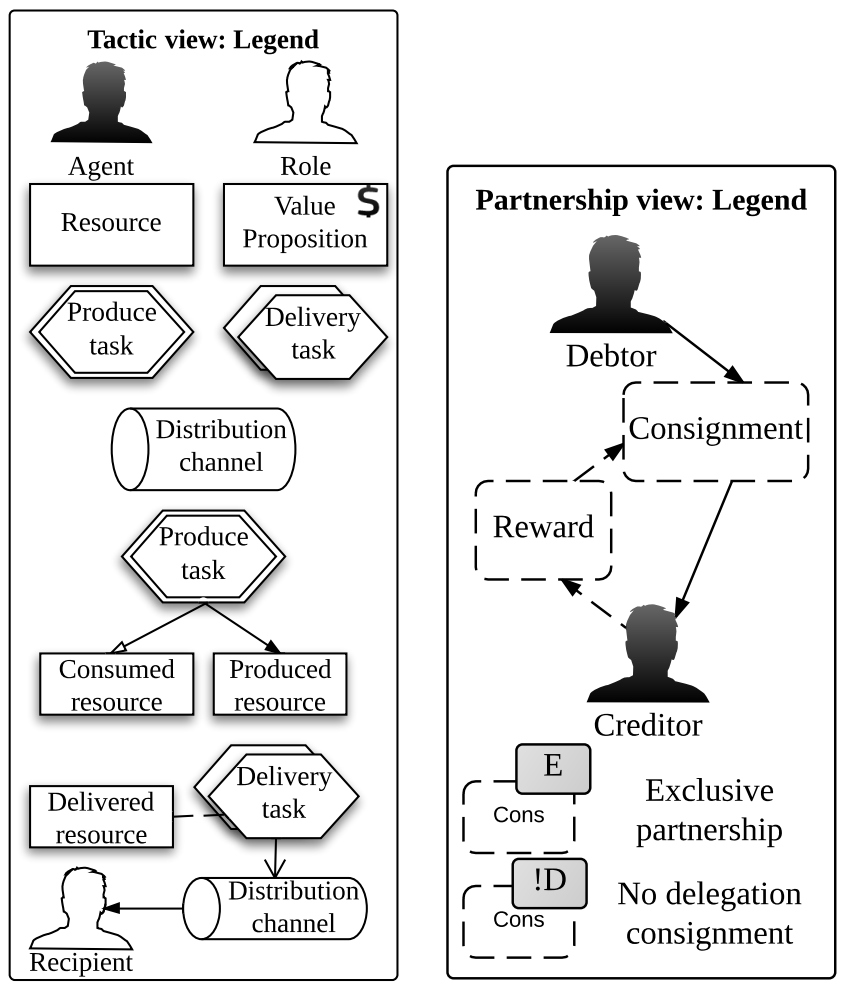

Fig. 1: Concepts in TBIM: (a) tactical view, and (b) partnership view

be used to evaluate the satisfaction of goals. One of the salient features of BIM is an extensive use of influence relationships that determine how the different elements in a model do impact one another. All details concerning BIM are in [10], [11].

TBIM consists of two complementary modeling views that enable a comprehensive representation of business tactics. The tactical view (Section IV-A) uses an extended version of BIM to describe the goals of the modeled organization as well as the high-level tactics to fulfill such goals. The partnership view (Section IV-B) represents a network of contractually-related organizational units.

\section{A. Tactical view}

Fig. 1(a) illustrates the graphical representation of the concepts in the tactical view, which are briefly described in the following paragraphs.

Agent and Role. As defined by Yu [28], an agent is an active entity that carries out actions to achieve goals by exercising its knowhow. Agents are intentional because their activities are motivated by the desire of attaining certain goals. A role is an abstract characterization of behaviors (also called "scripts" in a theatrical context) of a social agent within some specialized context or domain of endeavor. The scripts of a role are adopted by the agents that play that role. An actor is either a role or an agent. Graphically, agents and roles have an associated oval container that denotes the elements within the scope of that actor.

Resource and Value Proposition. To create value, a firm needs resources [24]. Resources can be any type of asset 
and include plants, equipment and cash reserves, patents, copyrights, reputation, brands and trade secrets, and people.

A value proposition is a particular kind of resource that a firm offers to a specific target customer segment. Value propositions differ from plain resources as they form the firm's primary source of revenue. Note that, in TBIM, the value proposition entity represents the value-holding resource that is provided to the customer segment, rather than the delivered value itself.

Produce Task. It is a set of activities devoted to the production of resources. This type of task consists not only in the execution of activities, but also contains working methods capable of producing outputs with specific features.

Delivery Task. It is concerned with the distribution of resources to agents and roles. This type of task deals with all the logistics of distribution of a product such as packaging, interaction with the customer, and efforts in making it available to the given target.

Distribution channel. It is a means, either virtual or physical, through which resources are delivered to customers. For companies, distribution channels are strategic assets with respect to reaching specific market segments.

Importantly, TBIM distinguishes between internal and external elements (such as Produce and Delivery Tasks, Resources and Distribution Channels), depending on whether they can be considered as part of the organization itself, or furnished by third party actors by mean of a partnership. Gray-colored figures depict elements that fall in this last category, hence provided by another actor.

\section{B. Partnership view}

The partnership view represents a collaboration network among actors. The primitive for modeling collaborations is that of commitment: a contractual agreement among actors on the execution of tasks, exchange of resources, and provision of distribution channels.

Commitments abstract away operational details [21], minimally constraining business executions. A commitment is a quaternary relation where a debtor actor commits to a creditor actor that a consignment will be delivered/provided, if (optionally) a reward is provided by the creditor.

Commitments relate elements that appear in the tactical view: debtor and creditor are agents and roles in the tactical view, while resources, tasks (of all types), and distribution channels constitute the consignment and the reward. As shown in Fig. 1, TBIM enriches commitments with two new attributes:

- Exclusivity. A commitment may be exclusive, meaning that the consignment shall be provided to that creditor only. This is useful to express the exclusive agreement contracts that are often employed among organizations.

- No delegation. A task referenced in a commitment can be delegable (by default) or not. If delegable, the debtor is authorized to delegate the execution of that task to another actor. This constraint-also used for security reasons [18]-represents the case in which a specific actor is trusted for a task, but only if the actor himself conducts the task.

\section{Evaluating Alternative Business Plans}

While TBIM allows for a high-level representation of business tactics, these need to be further refined in order to obtain an accurate evaluation and comparison. In [6], we have proposed a conceptual mapping between TBIM models and Business Process Modeling Notation (BPMN) models. The purpose of the mapping is to leverage on BPMN simulation techniques to evaluate the quality of different plans. We illustrate how business tactics can be automatically analyzed, either using a TBIM model or a corresponding set of BPMN processes.

- Evaluation of plans. Simulation techniques for BPMN can be used to evaluate alternatives TBIM business tactics in terms of execution time, usage of resources (human or material) and execution cost. In general, simulations enable estimating the quality of a plan quantitatively, and these quantities represent the expected effort that is necessary to carry out a tactic.

- Improvement of existing models. The outcome of BPMN simulations can be exploited as an input to improve TBIM tactics. This is done by conducting simulations for the different branches in a TBIM tactical view (introduced through OR-decompositions) or by evaluating alternative partnerships. The obtained results are compared and, based on the outcome, existing alternatives can be pruned, merged, or prioritized. For example, one could compare the off-line and online ticketing options to evaluate whether the MJF organization should better invest in both, or only in one of them.

- Partnerships maintenance. As pointed out by Telang et al. [21], «No enterprise is an island», for organizations need to exchange value with others to become and remain competitive. Partnerships should be continuously monitored and evaluated, in order to rely on the most effective network of partnerships. Business analysts can perform different analyses using the partnership view of TBIM: (i) Are all the resources and tasks in the current commitments actually used in the tactical view?; (ii) Is there an excessive fragmentation in suppliers which prevents economy of scale (e.g., multiple suppliers for similar goods)?; (iii) Are all external resources supported by at least one commitment in the partnership view?

- Identification of critical assets. Every organization relies on a set of core capabilities, the key enabler for a company to operate successfully. Business plans are centered on the creation of resources and the execution of activities that, together, constitute revenue for the company. While value propositions provide a direct stream of revenue for the organization, analysts are also interested in identifying the critical assets that, if not available, threaten the generation of value propositions. In the MJF scenario, artists represent a critical asset to protect, for there are no concerts 


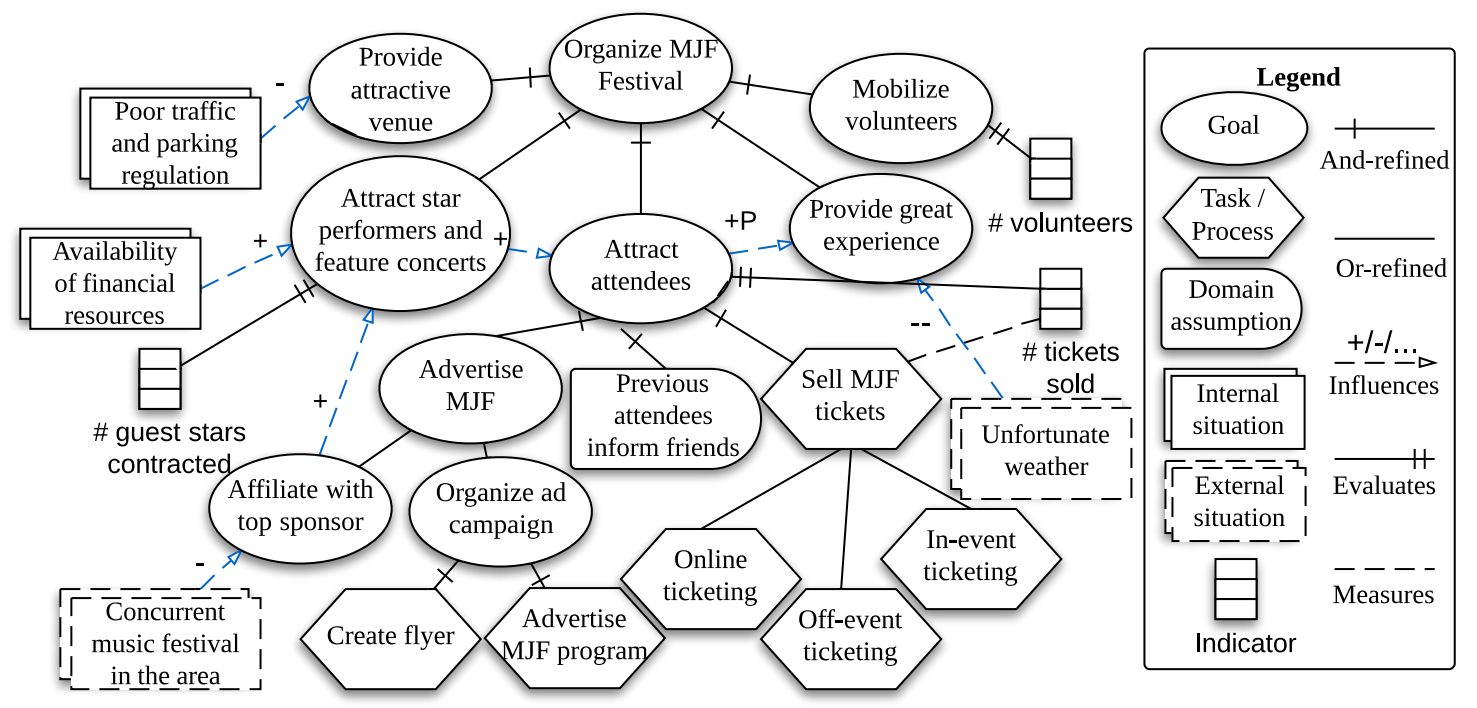

Fig. 2: Strategic modeling of the MJF organization case study with BIM

(this value proposition is threatened) if artists do not perform on stage.

\section{Modeling the MJF CASE Study}

We model the Montreux Jazz Festival organization based on the extensive description and representation through the business canvas that is provided in Osterwalder's doctoral dissertation [17]. In that work, the MJF is decomposed into a set of capabilities, each defining a set of related activities concerning the organization of an edition of the festival.

An initial version of our models is available in Francesconi's thesis [5]. This paper presents a revised, ameliorated, and polished version. Overall, it took us approximately twelve days to create the final version, which is the result of an incremental refinement process that went through multiple iterations. We started with an initial set nine goals and six tasks, which represented the top-level view on the MJF festival. Our refinement process led us to splitting the tactical view into three sub-domains counting $80+$ elements (goals, tasks, distribution channels, etc.) and 100+ relationships. Moreover, the partnership view counts 13 commitments among 13 actors for the provision of 35 elements.

We start our modeling with the BIM strategic model in Fig. 2. We do this because TBIM extends BIM with lower-level tactical elements: in other words, TBIM models are drawn as a refinement of BIM models. In Fig. 2, we represent the strategic goals, the external/internal situations that may affect the achievement of those goals, the indicators for measuring goal satisfaction and denial, and the high-level processes for fulfilling goals.

The top-level strategic goal is to Organize MJF Festival. To do so, five sub-goals are required: providing an attractive venue, attracting performers and featuring concerts, attracting attendees, providing a great experience, and mobilizing volunteers. Goals are eventually refined into processes; for example, Attract attendees is refined into process Sell MJF tickets, which can be performed either online, off-line, or through in-event ticketing. Some situations influence goal achievement. For example, the external situation Unfortunate weather threatens goal Provide great experience. The model shows measurable indicators, such as the number of volunteers, the number of tickets sold, and the number of guest stars contracted.

\section{A. Ticketing and Advertising}

Fig. 3 shows part of a TBIM tactical view for the capability concerning ticketing and advertising activities. This model extends the BIM model in Fig. 2 by further refining the goals Organize ad campaign (for advertising the festival) and Attract attendees (to foster participation) using the primitives offered by TBIM.

In order to advertise the festival and all of its events, the MJF organization decides to Create and distribute flyers to local people and to Distribute MJF program through different channels in order to reach both potential participants outside Switzerland as well as the attendees during the festival.

In order to Create and distribute flyers, the MJF organization decides to Print flyers through an external partnership (the task is gray-colored) which provides the resource Flyers that are printed on the provided Blank papers. The distribution of flyers can be carried out in two different manners: (a) the MJF organization can organize internally the distribution process, and rely on an external distribution channel by renting a Ticket shop; or (b) the organization may fully rely on an external partner to Distribute flyers via agencies and to let them to provide Local people with Flyers through Ticket agencies channels.

The second task for the MJF organizers is to Advertise MJF program. This task is refined into the execution of several delivery tasks, each concerned with a different aspect of the distribution. Distribute program during festival is carried out within the MJF organization in order to distribute the Concert program during the festival through their Event's stands with the intent of reaching Festival attendees. The program will also be distributed by external partners. Specifically, Radio 


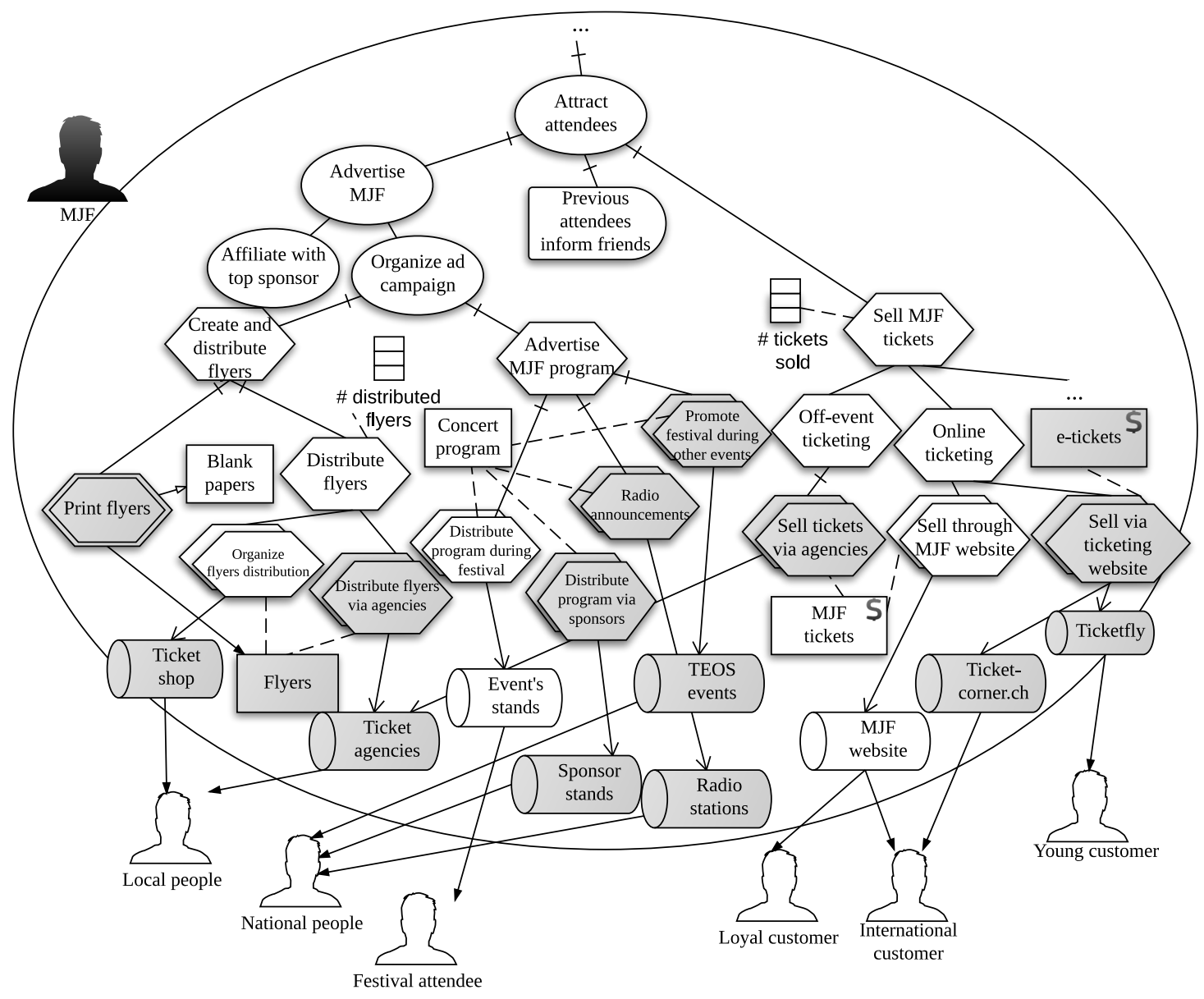

Fig. 3: Tactical view: ticketing and advertising capability

announcements are expected to be performed through Radio stations and the festival will be Promoted during other events such as the TEOS events. These two different delivery processes are intended to reach National people.

The part of the tactical view that refines goal Attract attendees shows alternative business tactics. The goal is refined into process Sell MJF tickets, which can be carried out in different non-exclusive ways, some of which are shown in the figure: Off-event ticketing and Online ticketing. The former is relying on an external partner that provides the Sell tickets via agencies delivery task, which requires MJF tickets and sells them via Ticket agencies. The latter task introduces further alternatives. Online ticketing can be done either through task Sell tickets through MJF website, or task Sell tickets via ticketing website. The first tactic enables selling tickets on the official MJF website, and the delivery of a hardcopy of the tickets by mail. The second tactic supports selling e-tickets via external partners' websites such as Ticket-corner.ch and Ticketfly. Tickets are value propositions for the MJF organization, for ticket sales constitute the $41 \%$ of the MJF festival incomes.

\section{B. Experience Delivery}

Another primary capability of the MJF organization is to deliver a unique experience to Festival attendees. The organization's plan is to manage the festival infrastructure, to
Place diverse shops \& food shops in the event location and to Coordinate and produce activities for a successful edition of the festival. The TBIM tactical model in Fig. 4 focuses on the distribution of merchandising and the provision of services to attendees. Specifically, the MJF plans to Sell merchandise and recordings through Event stands. The delivered resources are Merchandise and Live concerts recordings, which are value propositions for they form the $5 \%$ and the $4 \%$ of the overall revenue model, respectively.

Another important activity is to Advertise and sell of partners' products. This delivery task distributes Branded products via Event stands and Sponsor stands. The trade of branded products represents the $20 \%$ of festival revenues. Other tasks, such as Provide tattooing services and Deliver entertainment services are offered during the festival. Ultimately, International food, Local and typical food and Branded beverages are distributed to festival goers through the Distribute foods and beverages to attendees delivery task. Food and beverages (F\&B) form the $28 \%$ of the revenue model of the MJF. All these goods are made available to Festival attendees via the F\&B stands distribution channel.

\section{Concerts and Recordings}

Two additional objectives of the MJF organization are to Provide an attractive venue and to Attract star performers 


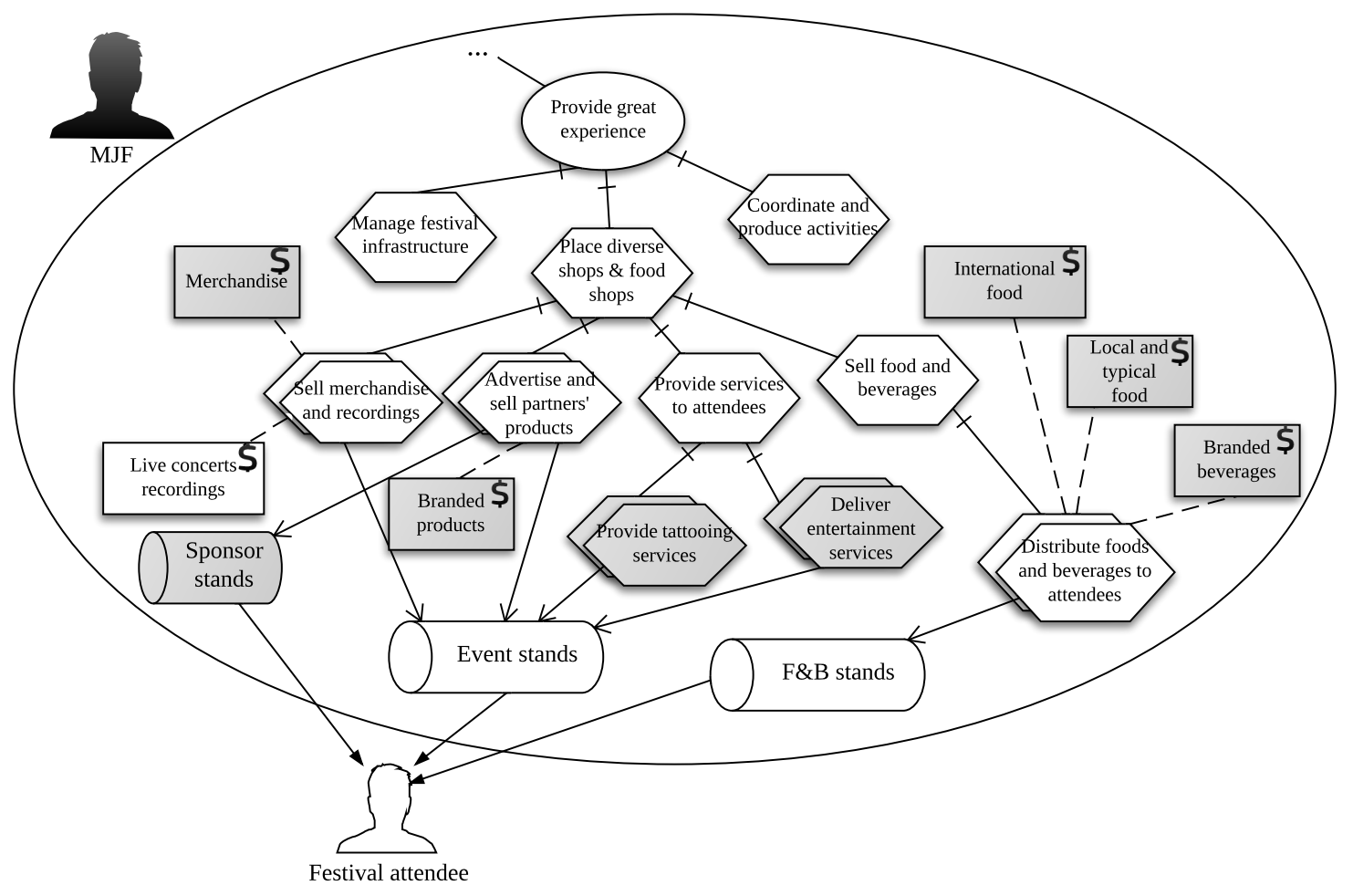

Fig. 4: Tactical view: experience delivery capability

and feature concerts. The first objective is refined into many aspects devoted in making the festival attractive to people. The organization, in way of achieving that, plans to Provide accessibility to the festival location, to Organize festival in charming location (specifically, between the mountains at the shores of the Lake of Geneva) and to get Funds raised. This latter is important because it defines the plans toward the production of one of the main source of revenue for the organization, the sale of concerts recordings, achieved by Producing live concerts recordings.

A key activity for the delivering concerts is to successfully Contract musicians and, thus, to Sign artists. This activity produces two different types of resources: Contracted star artists and Contracted ordinary artists; it does so by consuming Music artists (i.e., their availability is needed for the concert). Volunteers, Coordinators and Instrumentations, along with the aforementioned contracted artists, are all required resources in order to fulfill goal Produce concerts. The outcome of the production is a value proposition, Concerts, that is provided to Festival attendees and Radio listeners via different channels. The concerts, when the actors are performing on the stage, are being attended by festival goers at the foot of the Stage. An external partner is taking care of Live broadcasting the concerts by mean of Radio stations. Concerts are not only delivered live to attendees, they are also being recorded and processed in order to produce Live concerts recordings and Clips.

\section{Partnership View}

Fig. 6 illustrates the network of partnerships that concerns the Montreux Jazz Festival organization. As one could expect from a large-scale event like the MJF, there are several actors that are involved in the organization of the festival. To facilitate reading, we have omitted the rewards that the MJF organization shall return for the provision of the content of the commitments, whenever these rewards are money. While we acknowledge that modeling and analyzing monetary flows is important, this goes beyond the purpose of TBIM.

The partnership view shows how most auxiliary activities are delegated to external actors. For example, the catering actor takes care of the distribution of food and beverages to festival attendees. The festival organization does not have any expertise in recording and producing concerts. Thus, it relies on the Friends of the MJF association for the exclusive provision of Producers and Coordinators. These two resources are required, in combination with others, to produce concerts and recordings (see the tactical view in Fig. 5).

Activities such as printing tickets and flyers, as well as their distribution, are transferred to external partners such as Ticket agency, Ticketfly Corp and Ticketcorner.ch. The third partner is linked to the MJF organization through a commitment for selling e-tickets and MJF tickets via their ticketing website, with no right of delegation; this enforces the company to use their own portal to advertise and distribute tickets without relying on other companies. A Commercial unit rental agency is also offering to provide Ticket shop, which is used as a channel through which tickets are sold (see the tactical view in Fig. 3).

Notice that, for the MJF organization, artists are resources that are provided by Booking agencies. Artists are later then contracted (see Fig. 5) and become one of the primary assets for producing and providing concerts. 


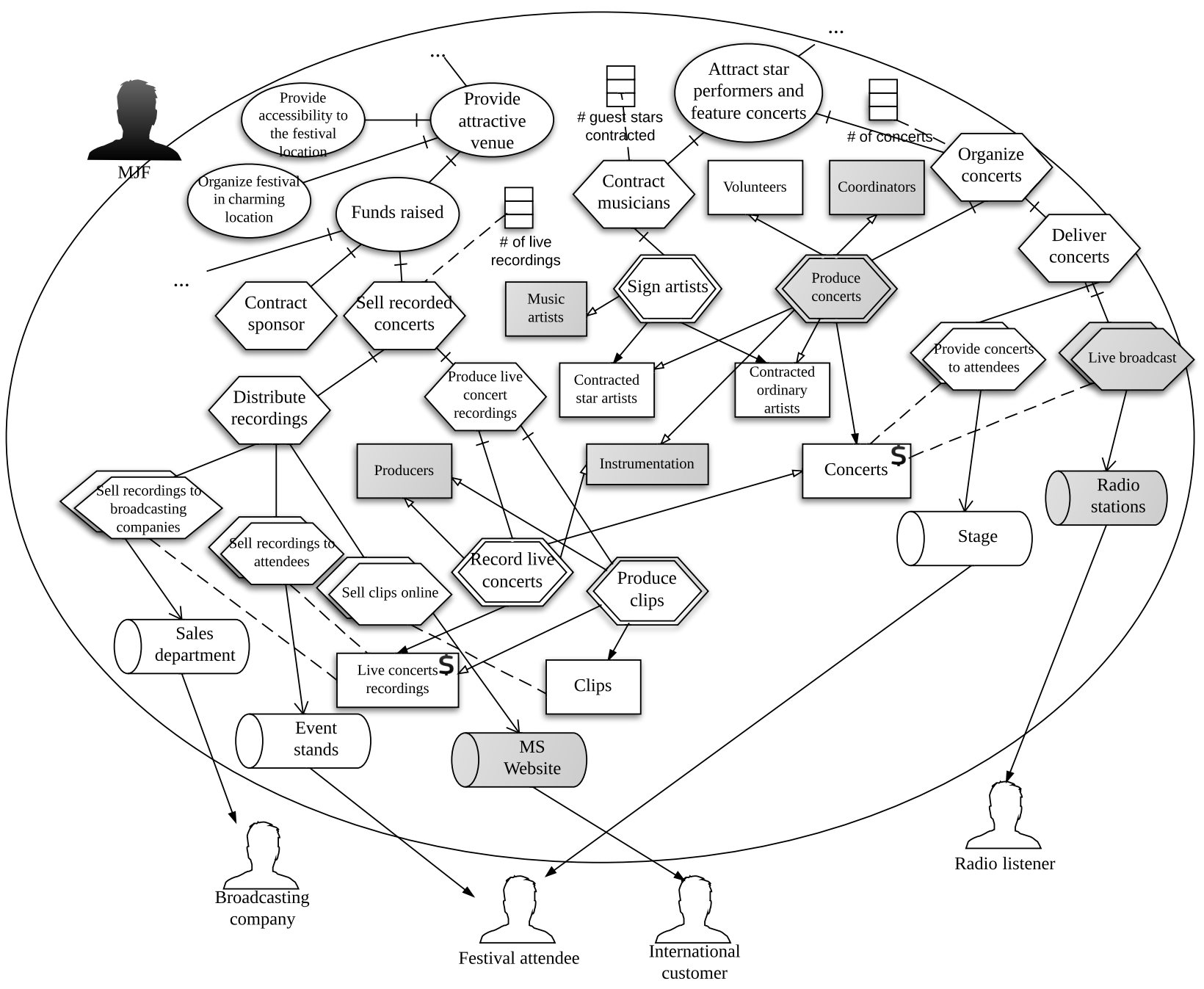

Fig. 5: Tactical view: concerts and recordings capability

\section{DISCUSSION}

In the previous section, we have shown TBIM models for the MJF case study. Our modeling exercise allowed us to explore several aspects of the organization, and TBIM has proven to be able to capture interesting details of the festival. In the following, we summarize strengths, limitations, and threats to validity.

\section{A. Strengths}

Expressiveness. Artists, one of the core assets for the modeled organization, are represented as resources. The language is expressive in that it highlights their importance by connecting them with activities that are expected to "consume" them (use their availability). This connection emphasizes the necessity of having contracted artists in order to create concerts.

Separation of concerns. TBIM decouples internal and external aspects of an organization in two views: the tactical view and the partnership view. This allows the modelers to focus on one perspective at a time, and to decouple the specification of a tactic from the choice of a suitable network of partnerships to fulfill the tactic. If adequately supported by a tool, this modeling style eases the creation of large models for organizations that rely on many external partners.

TBIM as a bridge. The larger an organization, the more complex and tangled are its business tactics. The MJF is a medium-sized case study that relies on several external organizations providing artists, infrastructures, goods and services. As we illustrated in the previous sections, TBIM models are moderately effective in dealing with large scenarios; while employing multiple actors and refinements through tree structures helps hierarchical modeling, the models may become clumsy and too large with representing very large settings. They can be used as an intermediate layer between high-level strategic models (e.g., BIM models or balanced scorecards) and low-level (large and complicated) operational models (e.g., BPMN models). TBIM models can be used as a guideline to organize BPMN models and can act as a traceability link between the operational and the strategic level. 


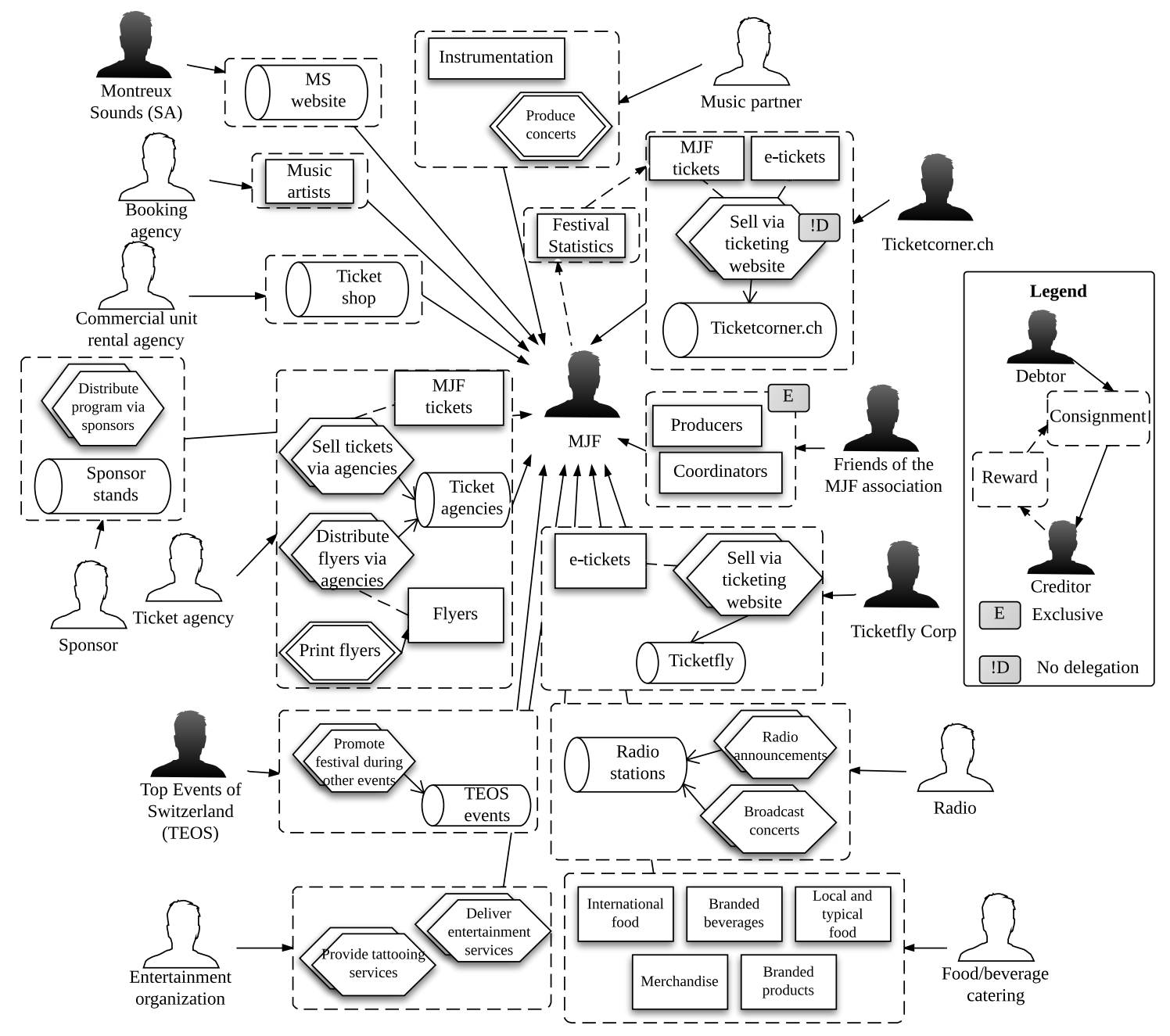

Fig. 6: MJF - Partnership view

\section{B. Limitations}

Money flow. TBIM offers poor support for modeling and analyzing money flows. Indicators, inherited from the BIM language, serve as quantitative evaluators that measures specific parameters. Thus, indicators can be used to monitor income and expenses. However, this approach does not provide a comprehensive tool to investigate revenue models, costs, rewards to partnerships, profits and and monetary losses. In the future, we need to investigate how to complement TBIM with value modeling approaches (e.g., [7]).

Contextual triggers. TBIM serves to define business tactics but does not provide a way to define triggers for the execution of (sub)plans when a specific set of events occurs, or to specify that a certain plan is not applicable under certain conditions. For instance, a company may want to define a set of backup plans to pursue when specific conditions are met. To such extent, we plan to investigate the usage of contextual annotations and context analysis [2].

No organizational structuring. Organizations are structured in different layers, each of which focuses on different aspects of the company and has specific duties and activities to pursue.
TBIM does not provide a way to decompose an organization in smaller units, departments and teams. TBIM needs to be complemented with primitives to express the structure within an organization.

\section{Threats to validity}

We report the main threats to validity that we encountered during the conduction of our research, and classify them in the four main categories proposed by Wohlin et al. [26]: conclusion, internal, construct, and external validity.

Conclusion validity concerns the ability to draw the correct conclusion. In our study, while the modeler is the person who knows the language best (making him adequate for the task at hand), he may also be misusing the language based on his tacit knowledge about the language, thereby leading to benefits that would not be achievable by others. Low statistical power is another threat; multiple modelers should be involved to obtain stronger conclusions.

Internal validity threats are about influences that affect the independent variable, without the researcher's knowledge. The modeler was also one of the authors of this paper; as such, this threat is not applicable. 
Construct validity is about the generalization of the results of the experiment. Our study suffers from mono-operation bias, for only one case study was considered in this research, and was conducted by a single subject.

External validity concerns conditions that limit the ability to generalize the results to industrial practice. To such extent, different case studies should be considered, for the obtained results apply only to the specific case study on the Montreaux Jazz Festival.

\section{RELATED WORK}

We review related work that provides alternative approaches for modeling enterprises and organizations.

Business ontologies. They suggest conceptual foundations for conceiving enterprises. Among the rich literature in the field, two prominent approaches are Uschold's enterprise ontology [22] and McCarthy's Resource/Event/Agent generalized accounting model [14]. The Business Motivation Model [16] is a standard that defines business plans by starting from the motivations of a company. These works provide sets of concepts (e.g., resources, duality, agents, strategy, activities, and motivations) that underlie several modeling languages, TBIM included.

Enterprise architectures. They provide principles, methods, and models for the design and realization of an enterprise. TOGAF [9] promotes a requirements-centered design of the architecture, which starts with a vision of the architecture, and includes governance and change management. The Zachman framework [29] models enterprises by filling in all the cells in a matrix where rows define the granularity level, and columns specify different aspects (why, when, what, how, where, who). These approaches do not offer a specific modeling language.

Value-based languages. They represent different aspects of a business enterprise. The $\mathrm{e}^{3}$ value [7] methodology models a network of enterprises creating, distributing, and consuming resources having an economic value. As observed by Andersson et al. [3], some concepts in BMO are similar to those of $\mathrm{e}^{3}$ value . Notably, the value-based approach is part of a current standardization activity that gave birth to the Value Delivery Modeling Language (VDML) [4]. Lo and Yu [13] suggest the usage of extended $i^{*}$ [27] agent- and goaloriented models to design collaborations-including resource exchange and task execution-among organizations. TBIM brings this notion further by suggesting different types of tasks (production, distribution), and uses commitments for relating business partners. $i^{*}$ and $\mathrm{e}^{3}$ value have been combined [8] to support e-service design. In their approach, the gaps between two models are filled in by the analyst. TBIM, instead, relies on a unified conceptual model.

Social commitments. They are relationships that tie together autonomous participants through declarative contracts [20]. Telang et al. [21] rely on commitments to propose an agent-oriented approach for specifying and verifying crossorganization business models. The notion of commitment is also part of the influential Agent-Object-Relationship (AOR) Metamodel [23], along with other notions such as agent, event, action, claim, and object. TBIM relies on a fine-grained ontology for both intentional elements and commitments that has been devised for business tactic modeling.

Business Process Modeling Languages. These approaches aim at the creation of models that represent the business processes within an organization, which are a major component of a business tactic. BPMN [25] is the de-facto standard notation, and it relies on the notions of activity and control flow. BPMN 2.0 [15] introduces support for the collaboration between different organizations through the collaboration and choreography diagrams. In previous work [6], we have proposed a mapping showing how to relate TBIM models to BPMN models.

\section{DISCUSSION AND OUTLOOK}

We have presented and discussed our modeling of the Montreux Jazz Festival organization case study using our proposed Tactical Business Intelligence Model, a conceptual modeling language that enables modeling and analysis for business tactics.

Our language combines high-level primitives for modeling organizational strategies (taken from the BIM language [11]) with notions for modeling business models (from the Business Model Ontology [17]). As discussed throughout the paper, the lessons learned from the modeling of a large-size case study include both strengths and weaknesses.

A strength of the language is that it clearly decouples the internal viewpoint (the tactic of the enterprise) from the partnership viewpoint (the network of relationships that enables realizing the tactic). This allows for systematic reasoning techniques concerning the fitness between partnerships and tactics. Moreover, when compared to the BMO modeling using the business canvas, TBIM comes with a more formal set of primitive concepts and relations between them.

A limitation is that it does not support quantitative moneyflow relationships that enable comparing alternative tactics from a cost-revenue standpoint. TBIM modeling can be complemented with techniques that support analyzing this aspect. Another limitation is that TBIM currently lacks of primitives to represent how organizations are internally structured. When compared to intuitive languages such as the business canvas, the learning curve for TBIM is steeper. Finally, the understandability of the modeling language has not been widely explored yet.

Our future work includes addressing these limitations, conducting further empirical studies with direct involvement of industrial partners, and developing a modeling and reasoning tool that can be used by end users.

\section{ACKNOWLEDGMENTS}

This work has been partially supported by the ERC advanced grant 267856 for a project titled "Lucretius: Foundations for Software Evolution" (http://www.lucretius.eu), and by the Natural Sciences and Engineering Research Council (NSERC) of Canada through the Business Intelligence Network. 


\section{REFERENCES}

[1] Derek.F. Abell. Defining the Business: the Starting Point of Strategic Planning. Prentice Hall, 1980.

[2] Raian Ali, Fabiano Dalpiaz, and Paolo Giorgini. A Goal-based Framework for Contextual Requirements Modeling and Analysis. Requirements Engineering, 15(4):439-458, 2010.

[3] Birger Andersson, Maria Bergholtz, Ananda Edirisuriya, Tharaka Ilayperuma, Paul Johannesson, Jaap Gordijn, et al. Towards a Reference Ontology for Business Models. In Proceedings of the International Conference on Conceptual Modeling (ER), pages 482-496. 2006.

[4] Arne Berre. Value Delivery Modeling Language (VDML). Technical report, Cordys Corporation B.V., 2012.

[5] Fabiano Francesconi. Modelling Business Plans with TBIM: a Language for Strategic Planning. Master's thesis, University of Trento, 2012. http://goo.gl/Z7XJE

[6] Fabiano Francesconi, Fabiano Dalpiaz, and John Mylopoulos. TBIM: A Language for Modeling and Reasoning about Business Plans. In Proceedings of the International Conference on Conceptual Modeling (ER), pages 33-46, 2013.

[7] Jaap Gordijn, Hans Akkermans, and Hans van Vliet. Designing and Evaluating E-business Models. IEEE Intelligent Systems, 16(4):11-17, 2001

[8] Jaap Gordijn, Eric Yu, and Bas van der Raadt. E-service Design using $\mathrm{i}^{*}$ and $\mathrm{e}^{3}$ value Modeling. IEEE Software, 23(3):26-33, 2006.

[9] The Open Group. TOGAF Version 9. The Open Group Architecture Framework. 2009.

[10] Jennifer Horkoff, Daniele Barone, Lei Jiang, Eric Yu, Daniel Amyot, Alex Borgida, and John Mylopoulos. Strategic Business Modeling: Representation and Reasoning. Software and Systems Modeling, pages $1-27,2012$

[11] Jennifer Horkoff, Alex Borgida, John Mylopoulos, Daniele Barone, Lei Jiang, Eric Yu, and Daniel Amyot. Making Data Meaningful: The Business Intelligence Model and Its Formal Semantics in Description Logics. In Proceedings of the International Conference on Ontologies, DataBases, and Applications of Semantics (ODBASE), pages 700-717. 2012

[12] Robert S. Kaplan and David P. Norton. Having Trouble with Your Strategy?: Then Map It. Harvard Business School Publishing Corporation, 2000

[13] Amy Lo and Eric Yu. From business models to service-oriented design: a reference catalog approach. In Proceedings of the International Conference on Conceptual Modeling (ER), pages 87-101, 2007.
[14] William E. McCarthy. The REA Accounting Model: A Generalized Framework for Accounting Systems in a Shared Data Environment. Accounting Review, 57(3):554, 1982.

[15] Object Management Group. Business Process Modeling Notation (BPMN) version 2.0. Technical report, 2006

[16] Object Management Group. Business Motivation Model Specification v1.1. Technical report, 2010.

[17] Alexander Osterwalder. The Business Model Ontology. PhD thesis, HEC Lausanne, 2004

[18] Elda Paja, Fabiano Dalpiaz, and Paolo Giorgini. Managing Security Requirements Conflicts in Socio-Technical Systems. In Proceedings of the International Conference on Conceptual Modeling (ER), volume 8217 of $L N C S$, pages 270-283, 2013.

[19] Ken Peffers, Tuure Tuunanen, Marcus A. Rothenberger, and Samir Chatterjee. A design science research methodology for information systems research. Journal of Management Information Systems, 24(3):4577, 2007.

[20] Munindar P. Singh. An Ontology for Commitments in Multiagent Systems: Toward a Unification of Normative Concepts. Artificial Intelligence and Law, 7:97-113.

[21] Pankaj R. Telang and Munindar P. Singh. Specifying and Verifying Cross-organizational Business Models: An Agent-oriented Approach. IEEE Transactions on Services Computing, 5(3):305-318, 2012.

[22] Mike Uschold, Martin King, Stuart Moralee, and Yannis Zorgios. The Enterprise Ontology. The Knowledge Engineering Review, 13(1):31-89, 1998.

[23] Gerd Wagner. The Agent-Object-Relationship Metamodel: Towards a Unified View of State and Behavior. Information Systems, 28(5):475504, 2003.

[24] B. Wernerfelt. A Resource-Based View of the Firm. Strategic Management Journal, 5(2):171-180, 1984.

[25] Stephen A. White and Derek Miers. BPMN Modeling and Reference Guide. Future Strategies Inc., 2008.

[26] Claes Wohlin, Per Runeson, Martin Höst, Magnus C Ohlsson, Björn Regnell, and Anders Wesslén. Experimentation in software engineering. Springer Science \& Business Media, 2012.

[27] Eric Yu. Modelling Strategic Relationships for Process Reengineering. $\mathrm{PhD}$ thesis, University of Toronto, 1996.

[28] Eric Yu. Towards Modeling and Reasoning Support for Early-Phase Requirements Engineering. In Proceedings of the International Requirements Engineering Conference (RE), pages 226-235, 1997.

[29] John A. Zachman. A Framework for Information Systems Architecture. IBM systems journal, 26(3):276-292, 1987. 